\title{
Critical point in the QCD phase diagram for extremely strong background magnetic fields
}

\section{Gergely Endrödi}

Institute for Theoretical Physics, Universität Regensburg, D-93040 Regensburg, Germany

E-mail: gergely.endrodi@physik.uni-r.de

ABSTRACT: Lattice simulations have demonstrated that a background (electro)magnetic field reduces the chiral/deconfinement transition temperature of quantum chromodynamics for $e B<1 \mathrm{GeV}^{2}$. On the level of observables, this reduction manifests itself in an enhancement of the Polyakov loop and in a suppression of the light quark condensates (inverse magnetic catalysis) in the transition region. In this paper, we report on lattice simulations of $1+1+1$-flavor QCD at an unprecedentedly high value of the magnetic field $e B=3.25 \mathrm{GeV}^{2}$. Based on the behavior of various observables, it is shown that even at this extremely strong field, inverse magnetic catalysis prevails and the transition, albeit becoming sharper, remains an analytic crossover. In addition, we develop an algorithm to directly simulate the asymptotically strong magnetic field limit of QCD. We find strong evidence for a first-order deconfinement phase transition in this limiting theory, implying the presence of a critical point in the QCD phase diagram. Based on the available lattice data, we estimate the location of the critical point.

Keywords: Lattice QCD, Lattice Gauge Field Theories, Lattice Quantum Field Theory, Phase Diagram of QCD

ARXIV EPRINT: 1504.08280 


\section{Contents}

1 Introduction 1

2 Setup and observables 3

$3 \quad$ Results at $e B=3.25 \mathrm{GeV}^{2} \quad 5$

4 Results in the asymptotic magnetic field limit 9

5 Conclusions $\quad 14$

A Effective action in the asymptotic magnetic field limit 16

$\begin{array}{ll}\text { B Simulating anisotropic pure gauge theory on the lattice } & 19\end{array}$

\section{Introduction}

Quantum chromodynamics (QCD) exhibits a finite temperature transition separating the chirally broken, low-temperature phase from the chirally symmetric, high-temperature regime, where quarks and gluons are deconfined. Although this transition is no real phase transition but merely an analytic crossover $[1,2]$, it is marked by the pronounced behavior of the corresponding (approximate) order parameters: the drop in the light quark condensates, accompanied by the increase in the Polyakov loop. The characteristic dependence of the transition temperature on further parameters of the system probes our understanding of QCD and maps out the phase diagram in the corresponding parameter space. One parameter that is thought to have rich physical applications, ranging from neutron star physics through heavy-ion collisions to the cosmology of the early universe, is a background (electro)magnetic field $B$. The relevant range of magnetic fields, where QCD interactions compete with the electromagnetic forces, is given by multiples of the pion mass squared $m_{\pi}^{2}$. We refer the reader to recent reviews on the subject [3-5].

QCD with background magnetic fields can be studied directly using non-perturbative lattice simulations. Continuum extrapolated results employing staggered quarks with physical masses have been used to map out the phase diagram [6] for $0 \leq e B<1 \mathrm{GeV}^{2}$. According to these results, the magnetic field increases the light quark condensates well below and well above $T_{c}$ (magnetic catalysis) but decreases them in the transition region [7] (inverse magnetic catalysis). As a result of this non-monotonous dependence of the condensate on $B$ and on $T$, the transition temperature is significantly reduced by the magnetic field. The same tendency has been observed for the Polyakov loop as well, giving a similarly decreasing transition temperature [8]. More recent lattice simulations employing different quark discretizations are consistent with this picture [9]. 
The magnetic catalysis of the condensate at low temperatures is a very robust concept. It arises naturally due to the Landau-level structure of charged particle energies in the presence of $B$. In the strong field limit, magnetic catalysis can be understood in terms of the dimensional reduction of the system and the high degeneracy of the lowest Landaulevel $[10,11]$. For low magnetic fields, it can be related to the positivity of the QED $\beta$ function that fixes the dependence of the condensate on $B$ to order $B^{2}[12,13]$. Magnetic catalysis even has connections to solid state physics models like the Hofstadter model [14]. In line with these arguments, the catalysis of the condensate at low temperatures was observed in a variety of model settings and effective theories of QCD. However, in most of these models, magnetic catalysis takes place not only for $T<T_{c}$, but for all temperatures, giving rise to a monotonously increasing dependence of $T_{c}$ on $B$. Thus, for the phase diagram, these models predict just the opposite of what the above discussed lattice results suggest. For a recent summary on these model approaches and a comparison to the lattice results, see refs. [4, 15].

While the mechanisms behind magnetic catalysis, as mentioned above, are quite transparent, the opposite behavior around $T_{c}$ - inverse magnetic catalysis - apparently has its origin in the rearrangement of the gluonic configurations that dominate the QCD path integral and is thus highly nontrivial [8]. Several attempts have been made recently to understand this behavior in effective approaches to QCD [16-33], among others, by introducing new, $B$-dependent model parameters or by taking into account the running of the QCD coupling with the magnetic field. Several of these models exhibit a non-monotonous $T_{c}(B)$ dependence, with an initial reduction followed by an enhancement due to the magnetic field. In certain settings, it was even shown that no matter how the existing parameters of the model are tuned as functions of $B$, the transition temperature always tends to rise above a given threshold magnetic field [34].

It is just the apparent universality of magnetic catalysis that has made the lattice results about inverse catalysis and the decreasing $T_{c}(B)$ dependence for $0 \leq e B<1 \mathrm{GeV}^{2}$ so unexpected. It was speculated that magnetic catalysis should reappear at even stronger magnetic fields, and different hypotheses were recently put forward about the strong $B$ regime of the phase diagram. In particular, the strong $B$ limit was argued to induce a new critical point [35]. The transition temperature was conjectured to turn around and increase if the magnetic field is sufficiently strong [36-39]. In other cases, $T_{c}$ was argued to keep decreasing and to hit zero [40]. The transition temperatures for chiral restoration and for deconfinement were predicted to split [41] in the presence of the magnetic field, and a splitting between the chiral restoration temperature for the up and down quarks was also argued to take place [39, 42]. Let us refer the reader to the recent reviews [4, 15] for details.

In this paper, we aim to check these conjectures by means of first-principles lattice simulations of $1+1+1$-flavor QCD at an unprecedentedly strong magnetic field $e B=3.25 \mathrm{GeV}^{2}$. In addition, we also simulate the $B \rightarrow \infty$ limit directly, by considering the effective theory relevant for this limit $[43,44]$. We find strong evidence that this limiting theory has a first-order deconfinement phase transition and, thus, the QCD phase diagram exhibits a critical point at strong magnetic fields, where the analytic crossover terminates. Based on our results, we estimate the location of the critical point, and sketch 
the dependence of the deconfinement transition temperature on $B$ over a broad range. Besides answering a fundamental question about the QCD phase diagram, we believe that the results will also be useful for building/refining effective theories and models of QCD.

The paper is organized as follows. In section 2 we discuss the details of the simulations and define the observables used to study the phase diagram. Section 3 contains the lattice results in ordinary QCD, followed by section 4, where we discuss the simulations of the anisotropic theory in the asymptotic limit. The derivation of this effective theory and the employed simulation algorithms are discussed in the appendices appendix A and appendix B. Finally, in section 5 we summarize our findings regarding the QCD phase diagram and conclude.

\section{Setup and observables}

We consider a spatially symmetric $N_{s}^{3} \times N_{t}$ lattice with spacing $a$ so that the temperature is given by $T=\left(N_{t} a\right)^{-1}$, the spatial volume by $V=\left(N_{s} a\right)^{3}$ and the four-volume by $V_{4}=V / T$. Given this geometry, we simulate $1+1+1$-flavor QCD, described by the partition function,

$$
\mathcal{Z}=\int \mathcal{D} U e^{-\beta S_{g}} \prod_{f=u, d, s}\left[\operatorname{det} M\left(U ; a^{2} q_{f} B, m_{f} a\right)\right]^{1 / 4},
$$

given by the functional integral over the gluonic links $U$. We employ stout smeared rooted staggered quarks described by the fermion matrix $M$. In eq. (2.1), $S_{g} \equiv s_{g} V_{4}$ is the tree-level Symanzik improved gauge action and $\beta=6 / g^{2}$ the inverse gauge coupling. For further details of the simulation setup and algorithm, see refs. [6, 45]. The parameters of the fermion matrix are the quark masses $m_{u}=m_{d} \neq m_{s}$ and the electric charges $q_{u}=-2 q_{d}=-2 q_{s}=2 e / 3(e>0$ is the elementary charge), which enter in the product with $B$. The quark masses are set to their physical values along the line of constant physics [46]. The magnetic field is oriented along the positive $z$-direction and has the quantized flux

$$
\Phi \equiv\left(a N_{s}\right)^{2} e B=6 \pi N_{b}, \quad N_{b} \in \mathbb{Z}, \quad 0 \leq N_{b}<N_{s}^{2},
$$

where we used that the smallest charge in the system is that of the down quark. Lattice discretization effects are suppressed as long as the flux quantum $N_{b}$ is much smaller than the period $N_{s}^{2}$. Previous experience suggests that $N_{b}<N_{s}^{2} / 16$ is a reasonable choice [6].

In the following, we employ the fixed- $N_{t}$ approach and change the temperature $T(\beta)=$ $\left(N_{t} a(\beta)\right)^{-1}$ by varying the inverse gauge coupling. This also implies that a given flux quantum corresponds to different magnetic fields at different temperatures, i.e. $e B \propto N_{b} T^{2}(\beta)$. In particular, we choose $\beta$ values where a fixed magnetic field $e B=3.25 \mathrm{GeV}^{2}$ is represented by integer flux quanta. Although this implies that only discrete temperatures are allowed, at this strong magnetic field the temperature differences are small enough in order to map out the transition region (see below). 
Next, we define the observables that can be used to pin down the transition temperature. We begin with the quark condensates and susceptibilities, signaling chiral symmetry,

$$
\left\langle\bar{\psi} \psi_{f}\right\rangle \equiv \frac{1}{V_{4}} \frac{\partial \log \mathcal{Z}}{\partial m_{f}}, \quad\left\langle\chi_{f}\right\rangle \equiv \frac{\partial\left\langle\bar{\psi} \psi_{f}\right\rangle}{\partial m_{f}}
$$

and employ the normalization inspired by the Gell-Mann-Oakes-Renner relation, introduced in ref. [7] for the condensate,

$$
\begin{aligned}
\Sigma_{u, d}(B, T) & =\frac{2 m_{u d}}{M_{\pi}^{2} F^{2}}\left[\left\langle\bar{\psi} \psi_{u, d}\right\rangle_{B, T}-\left\langle\bar{\psi} \psi_{u, d}\right\rangle_{0,0}\right]+1, \\
\chi_{u, d}^{\Sigma}(B, T) & =\frac{2 m_{u d}^{2}}{M_{\pi}^{2} F^{2}}\left[\left\langle\chi_{u, d}\right\rangle_{B, T}-\left\langle\chi_{u, d}\right\rangle_{0,0}\right] .
\end{aligned}
$$

Here, $M_{\pi}=135 \mathrm{MeV}$ is the pion mass and $F=86 \mathrm{MeV}$ the chiral limit of the pion decay constant. Both $\Sigma$ and $\chi^{\Sigma}$ are free of additive and of multiplicative divergences [6]. In addition, $\Sigma$ is normalized to be unity at $T=B=0$ and approaches zero above the transition region [7]. The vacuum values necessary for the additive renormalization were determined in refs. $[6,7]$.

The approximate order parameter for center symmetry, related to the deconfinement transition, is the Polyakov loop, defined on the lattice as

$$
P=\frac{1}{V}\left\langle\sum_{\mathbf{x}} \operatorname{Tr} \prod_{t} U_{4}(t, \mathbf{x})\right\rangle .
$$

In full QCD, the fermion determinant breaks center symmetry explicitly, so that the spontaneous breaking always occurs towards the real center element and $\langle\operatorname{Re} P\rangle$ is a valid (approximate) order parameter. In pure gauge theory (this will be relevant for the $B \rightarrow \infty$ limit, see section 4), there is no explicit breaking and the three center sectors are equivalent. In this case, it is convenient to consider the projection of the Polyakov loop to the nearest center element (see, e.g., ref. [47]),

$$
P^{\mathrm{pr}}= \begin{cases}\operatorname{Re} P, & \arg P \in[-\pi / 3, \pi / 3], \\ \operatorname{Re}\left[P e^{-i 2 \pi / 3}\right], & \arg P \in(\pi / 3, \pi], \\ \operatorname{Re}\left[P e^{i 2 \pi / 3}\right], & \arg P \in(-\pi,-\pi / 3) .\end{cases}
$$

Simulating pure gauge theory on a finite lattice, $\langle P\rangle$ always vanishes due to the tunneling between center sectors, while $\left\langle P^{\mathrm{pr}}\right\rangle$ is positive in the deconfined phase. The susceptibility of the projected Polyakov loop is defined as

$$
\chi_{P^{\mathrm{pr}}}=V\left[\left\langle P^{\mathrm{pr} 2}\right\rangle-\left\langle P^{\mathrm{pr}}\right\rangle^{2}\right]
$$

The Polyakov loop renormalizes multiplicatively, with a temperature-dependent renormalization constant

$$
P_{r}(T, B)=Z(T) \cdot P(T, B)
$$


which is determined by enforcing $\left\langle P_{r}\left(T_{\star}, 0\right)\right\rangle=P_{\star}$ and we chose $T_{\star}=162 \mathrm{MeV}$ and $P_{\star}=1$. The renormalization was discussed in detail and $Z(T)$ was determined in ref. [8]. Notice that while $\operatorname{Re} P<3$ by construction, the renormalized observable has no upper bound.

An observable that strongly correlates with $P$ - and, thus, is sensitive to the deconfinement transition - is the strange quark number susceptibility,

$$
c_{2}^{s}=\frac{1}{V_{4} \cdot T^{2}} \frac{\partial^{2} \log \mathcal{Z}}{\partial \mu_{s}^{2}},
$$

Note that $c_{2}^{s}$ contains neither additive nor multiplicative divergences.

Finally, the trace anomaly

$$
I^{(\Phi)}=-\left.\frac{1}{V_{4}} \frac{\partial \log \mathcal{Z}}{\partial \log a}\right|_{\Phi}
$$

can be written as a sum of gluonic, fermionic and magnetic contributions [13],

$$
I^{(\Phi)}(B, T)=\frac{\partial \beta}{\partial \log a}\left\langle s_{g}\right\rangle-\sum_{f} \frac{\partial\left(m_{f} a\right)}{\partial \log a}\left\langle\bar{\psi} \psi_{f}\right\rangle+b_{1}(e B)^{2},
$$

where $b_{1}=\sum_{f}\left(q_{f} / e\right)^{2} /\left(4 \pi^{2}\right)$ is the lowest-order QED $\beta$-function coefficient. The magnetic term appears due to electric charge renormalization and stems from the counter-term canceling the $B$-dependent additive divergence of the thermodynamic potential $\log \mathcal{Z}$ [13]. Note that this term is finite and independent of the regularization, once the continuum limit is taken, see discussion in ref. [13]. Notice furthermore that the derivative in the definition of $I$ is evaluated at fixed magnetic flux $\Phi$ and not at fixed magnetic field $e B$ [this is indicated by the superscript $(\Phi)$ ]. The need for distinguishing between the two directional derivatives was first discussed in ref. [48] and put into practice for the trace anomaly in ref. [13].

\section{Results at $e B=3.25 \mathrm{GeV}^{2}$}

We extend the previously published data on the light condensates and susceptibilities [7], on the Polyakov loop [8], on the strange quark number susceptibility [6], and on the trace anomaly $[13,48]$ using our new results at $e B=3.25 \mathrm{GeV}^{2}$. To achieve this magnetic field strength, a temporal lattice extent $N_{t}=16$ turned out to be necessary. These $N_{t}=16$ lattices are finer than the finite temperature configurations used in refs. [6-8] $\left(N_{t}=6,8\right.$ and 10) to extrapolate to the continuum limit. Thus, our results - although not strictly continuum extrapolated - are expected to lie close to the limit $a \rightarrow 0$. We use two spatial lattice sizes $32^{3} \times 16$ and $48^{3} \times 16$ to control finite size effects.

Let us start the discussion with the light quark condensates. The average of $\Sigma_{u}$ and $\Sigma_{d}$ is plotted in the left panel of figure 1 , compared to the $B=0$ and $B=1 \mathrm{GeV}^{2}$ continuum extrapolated results [7]. In addition to the data at nonzero temperatures, we also indicate an estimate for the zero-temperature condensate. This is obtained by fitting and extrapolating the available lattice data at $T=0$ by a free-theory inspired form $\sim B \log B$. The systematic uncertainty is taken into account by varying the fit interval. 

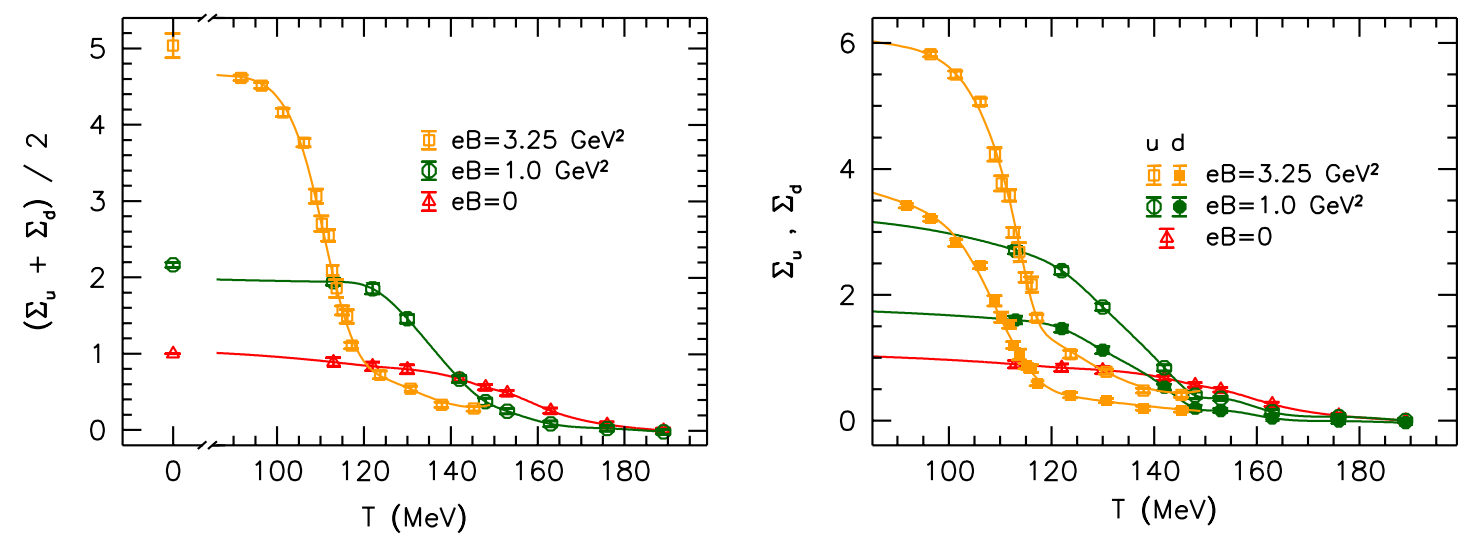

Figure 1. Left panel: average light quark condensate as a function of the temperature for three different magnetic fields. Right panel: up (open points) and down (filled points) quark condensates for the same set of magnetic fields. The curves are spline interpolations and merely serve to guide the eye.

While the condensate is increased by the magnetic field at low temperatures, reflecting the well-known magnetic catalysis effect, the results also clearly show the reduction of $\Sigma_{u}+\Sigma_{d}$ in the transition region. Thus, inverse magnetic catalysis is observed to persist in the transition region even for our strong magnetic field $e B=3.25 \mathrm{GeV}^{2}$, pushing $T_{c}$ further down. In particular, we employ the inflection point of the average condensate to find $T_{c}\left\{\Sigma_{u d}\right\}=112(3) \mathrm{MeV}$. As a side-remark, we mention that since the transition region is shifted to considerably lower temperatures, the vacuum values determined for $3.45<\beta<3.85$ in refs. [6, 7] suffice to perform the additive renormalization of the condensates, and there is no need for additional $T=0$ simulations on finer lattices.

Due to the different electric charges, $\Sigma_{u}$ is expected to be more sensitive to the magnetic field than $\Sigma_{d}$. On that account, even a splitting in the transition temperatures might seem plausible, see refs. [39, 42]. To check whether this is the case, in the right panel of figure 1 the two condensates are plotted separately. Even though the difference $\Sigma_{u}-\Sigma_{d}$ is pronounced throughout the temperature range in question, fitting for the inflection points gives consistent values $T_{c}\left\{\Sigma_{u}\right\}=112(3) \mathrm{MeV}$ and $T_{c}\left\{\Sigma_{d}\right\}=111(3) \mathrm{MeV}$. An apparent implication of this finding is that the temperature, at which the transition between the chirally broken and restored phases takes place, is encoded in the gluonic configurations rather than in the operator insertion. In lattice language; $T_{c}$ seems to be a quantity driven predominantly by sea and not by valence effects. This also suggests that purely gluonic observables would also exhibit similar transition temperatures.

This brings us to the simplest, purely gluonic quantity: the Polyakov loop (2.5). The (real part of the) renormalized observable is plotted in the left panel of figure 2, for the same set of magnetic fields, and is observed to be drastically enhanced by the magnetic field for all temperatures. The inflection point of $P_{r}(T)$ is much more pronounced as compared to the case at $B=1 \mathrm{GeV}^{2}$ and is determined to be $T_{c}\{P\}=109(3) \mathrm{MeV}$. This value is indeed consistent with the transition temperatures obtained above for the 

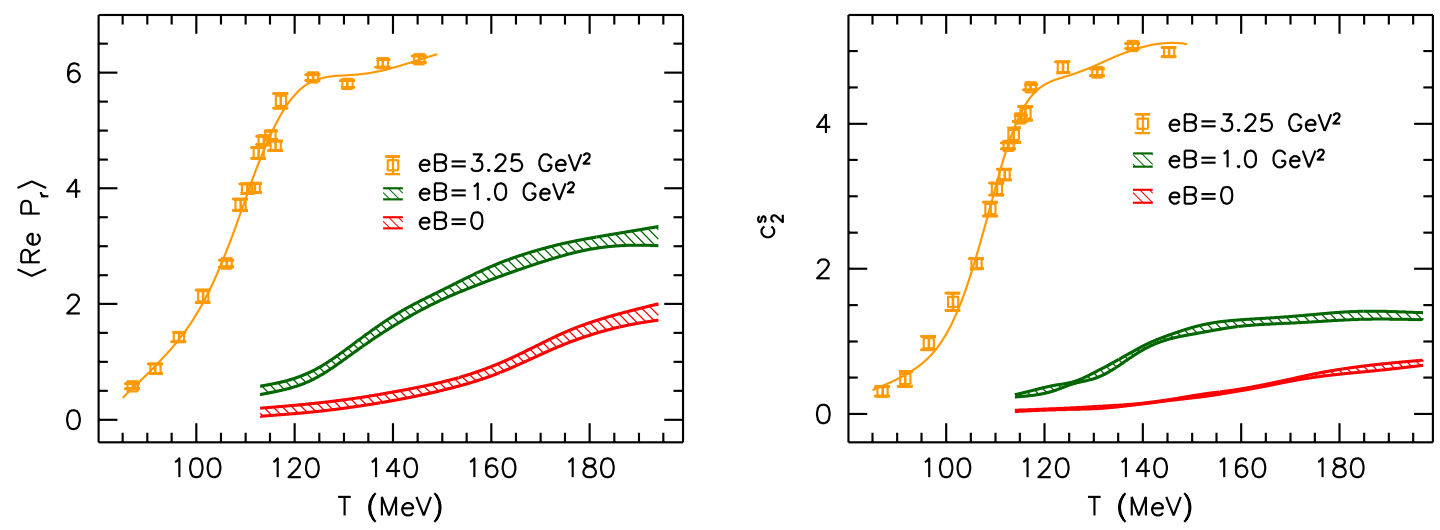

Figure 2. Left panel: the Polyakov loop for three values of $e B$. At the highest magnetic field, the curve is a spline interpolation, while for the lower fields the band is the result of a combined continuum extrapolation and interpolation in $T$ [8]. Right panel: the strange quark number susceptibility for the same set of magnetic fields. For the highest magnetic field, a spline interpolation is shown, whereas for the lower fields the bands represent a continuum estimate based on the results of ref. [6].

light quark condensates. We conclude that the gluonic configurations are vastly different on the two 'sides' of the transition, and predestine the behavior of the light condensates, independently of the electric charge that appears in the operator. We also observe the strange quark number susceptibility to exhibit an analogous trend, see the right panel of figure 2. Performing a similar fit as for $P_{r}$, we obtain $T_{c}\left\{c_{2}^{s}\right\}=109(3) \mathrm{MeV}$.

A further observable of interest for the QCD equation of state is the trace anomaly (2.10). It measures the breaking of conformal symmetry by the gluonic condensate, by the quark condensates and by the magnetic field itself. As $B$ grows, the latter effect becomes dominant and $I^{(\Phi)}$ is increased drastically, as visible in figure 3 . Since $I^{(\Phi)}$ contains $B$-dependent contributions already at zero temperature, the usual normalization $I^{(\Phi)} / T^{4}$ is not useful [13]. This large $T=0$ contribution also damps the behavior of $I^{(\Phi)}$ in the transition region. The small kink around $T_{c}$, moving towards smaller temperatures as $B$ grows, is to some extent still visible. We note that in order to determine further quantities related to the equation of state (e.g. pressure, entropy density etc.), one would need ad-

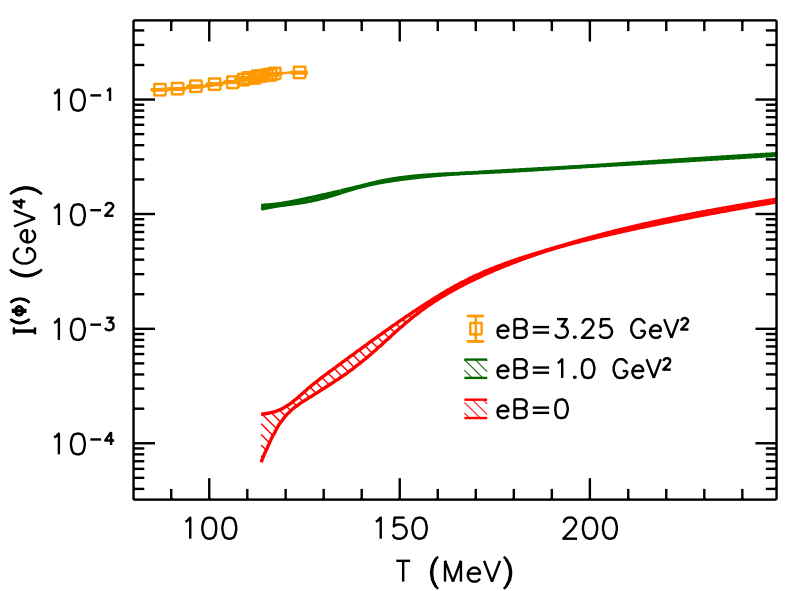

Figure 3. The trace anomaly for three different magnetic fields. Note the logarithmic scale. 

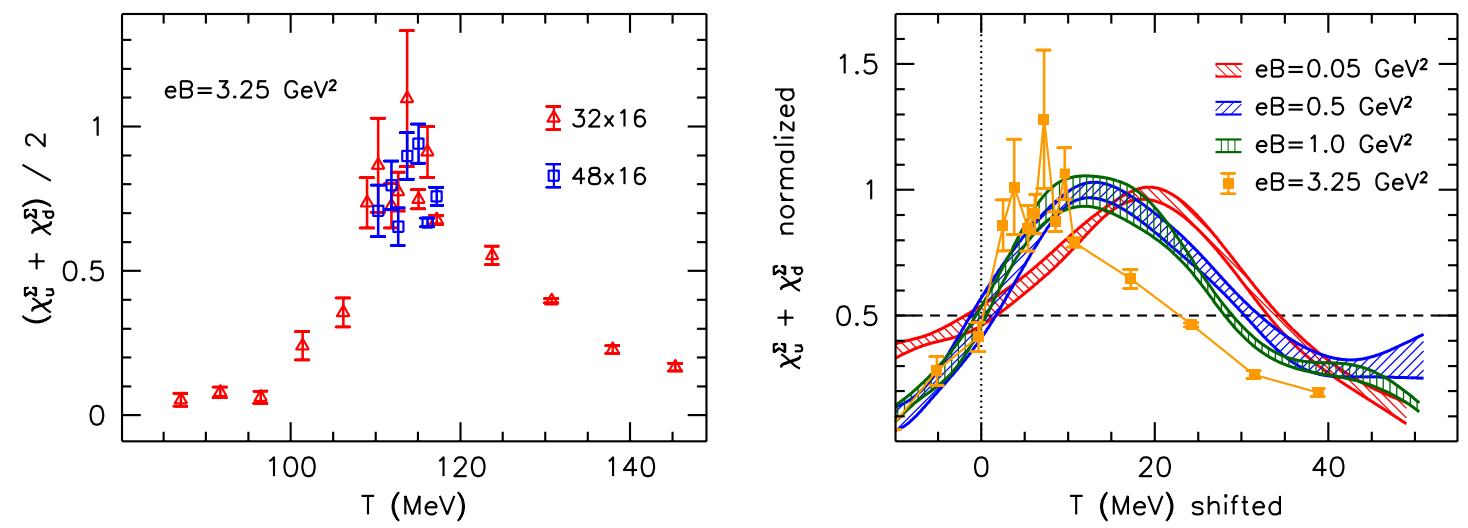

Figure 4. Left panel: finite size scaling of the average light quark susceptibility. Right panel: the dependence of the peak width on the magnetic field.

ditional simulations at low temperature (see the method developed in ref. [13]). This is outside the scope of the present paper.

Besides the characteristic temperature, the strength of the transition at high magnetic fields is also of interest. To determine, whether the smooth crossover at $e B<1 \mathrm{GeV}^{2}$ turns into a real phase transition at $e B=3.25 \mathrm{GeV}^{2}$, we analyze the average of the light quark susceptibilities $\chi_{u}^{\Sigma}+\chi_{d}^{\Sigma}$. This observable exhibits a peak at the transition temperature, see the left panel of figure 4 . For real phase transitions, the height $h$ of this peak diverges in the infinite volume limit: $h \propto V$ for first-order transitions and $h \propto V^{\alpha}$ with a critical exponent $\alpha<1$ for second-order transitions. In contrast, for the case of an analytic crossover, $h$ is independent of the volume. To perform this finite size scaling study, we compare the results obtained on the $48^{3} \times 16$ and on the $32^{3} \times 16$ ensembles. The left panel of figure 4 shows no sign of a singularity as $V$ is increased (note that for a first-order transition, the peak heights for the two volumes would differ by more than three). This leads us to conclude that the transition remains an analytic crossover even at $e B=3.25 \mathrm{GeV}^{2}$. We mention moreover that finite volume effects are also absent from the other observables discussed above.

Although the transition remains an analytic crossover, it is instructive to analyze the $B$-dependence of the susceptibility peak in more detail. We normalize $\chi_{u}^{\Sigma}+\chi_{d}^{\Sigma}$ such that its peak maximum equals unity, and plot it in the right panel of figure 4 against the temperature. Here, $T$ is shifted so that the observable equals 0.5 at zero. Then, the peak width $w(B)$ at half maximum can be read off at the rightmost intersection of the observable with 0.5. Clearly, $w(B)$ decreases as $B$ grows, signaling that the transition becomes stronger in the presence of the magnetic field. We will return to this observation below in section 5 .

Besides being useful for quantifying the strength of the transition, the peak of the susceptibility allows for yet another determination of the transition temperature. Fitting for the peak maximum, we obtain $T_{c}\left\{\chi_{u d}^{\Sigma}\right\}=113(4) \mathrm{MeV}$, consistent with the results obtained for all other observables. We mention that the peak positions for the up and down quark susceptibilities also agree within errors. 


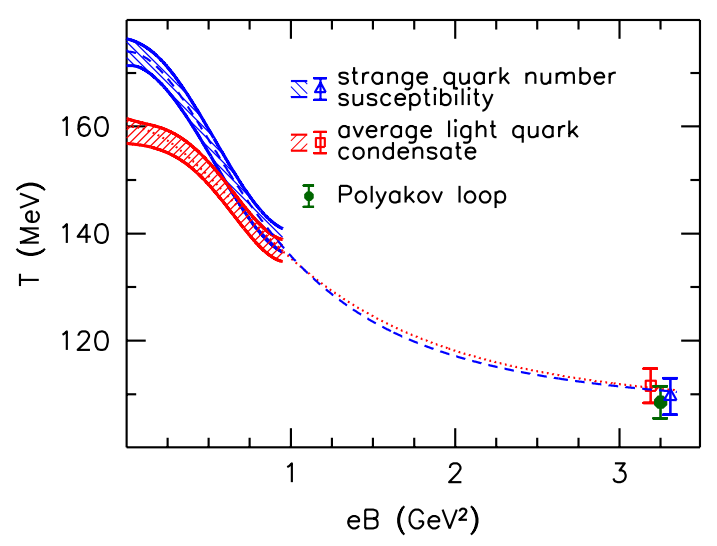

Figure 5. The QCD phase diagram in the magnetic field-temperature plane. Previous results at weaker magnetic fields [6] are complemented by our findings at high $e B$. The points have been slightly shifted horizontally for better visibility. The dotted and the dashed lines show an interpolation of the results for $\Sigma_{u}+\Sigma_{d}$ and for $c_{2}^{s}$, respectively, according to eq. (3.1).

\begin{tabular}{|c|c|c|c|}
\hline & $T_{c}(0)$ & $a_{1}$ & $a_{2}$ \\
\hline$\Sigma_{u d}$ & $160(2) \mathrm{MeV}$ & $0.54(2)$ & $0.82(2)$ \\
\hline$c_{2}^{s}$ & $174(2) \mathrm{MeV}$ & $0.78(1)$ & $1.28(1)$ \\
\hline
\end{tabular}

Table 1. Fit parameters of the function (3.1).

Finally, in figure 5 we summarize our determinations of $T_{c}$ in the QCD phase diagram. We consider the results for $e B<1 \mathrm{GeV}^{2}$ obtained for the light quark condensates and for the strange quark number susceptibility [6]. In addition, we also include the transition temperatures at $e B=3.25 \mathrm{GeV}^{2}$ obtained using the light quark condensates, the strange quark number susceptibility and the Polyakov loop. (Note that the inflection point of $P_{r}$ at $e B<1 \mathrm{GeV}^{2}$ is not pronounced enough to allow for a stable fit.) To interpolate $T_{c}\left\{\Sigma_{u d}\right\}$ and $T_{c}\left\{c_{2}^{s}\right\}$ for all magnetic fields, we found the following function sufficient,

$$
T_{c}(e B)=T_{c}(0) \cdot \frac{1+a_{1}(e B)^{2}}{1+a_{2}(e B)^{2}},
$$

giving the fit parameters shown in table 1 . The resulting fit is also shown in the figure.

\section{Results in the asymptotic magnetic field limit}

Our results in the right panel of figure 4 indicate that the transition becomes significantly sharper as the magnetic field increases. This observation raises the question: what happens if $B$ is even larger? Does the crossover terminate and turn into a real phase transition? To answer this question, we have to consider the limit $e B \gg \Lambda_{\mathrm{QCD}}^{2}$. Asymptotic freedom dictates that in this limit quarks and gluons decouple from each other. Still, the explicit breaking of rotational symmetry by $B$ and the corresponding dimensional reduction in the quark sector [10] suggests that this limit is not simply given by a pure gluonic theory 
plus non-interacting (electrically charged) quarks. Indeed, based on the structure of the gluon propagator in strong magnetic fields, ref. [43, 44] has shown that the effective action describing this limit is an anisotropic pure gauge theory. The anisotropy amounts to an enhancement of the chromo-dielectric constant in the direction parallel to the magnetic field, characterized by the coefficient $\kappa$,

$$
e B \gg \Lambda_{\mathrm{QCD}}^{2}: \quad \mathcal{L}=\frac{1}{g^{2}}\left[\operatorname{tr} \mathcal{B}_{\|}^{2}+\operatorname{tr} B_{\perp}^{2}+\left(1+g^{2} \kappa(B)\right) \operatorname{tr} \mathcal{E}_{\|}^{2}+\operatorname{tr} \mathcal{E}_{\perp}^{2}\right], \quad \kappa(B) \propto B .
$$

The definition of the gluonic field strength components $\mathcal{B}$ and $\mathcal{E}$ is given in eq. (A.2) below. The enhancement of the parallel chromo-dielectric constant implies that the corresponding field strength component $\mathcal{E}_{\|}$is suppressed. This tendency is already visible in our full QCD simulations at strong magnetic fields, see figure 11 in appendix A below.

Therefore, as $B$ is increased, the QCD effective Lagrangian approaches the anisotropic gauge theory given by eq. (4.1). Assuming that this theory has a first-order phase transition, ref. [35] has conjectured that the strong magnetic field region of the QCD phase diagram should exhibit a critical point. Here we address this question in more detail. First of all, in appendix A, we reproduce the results of ref. [43, 44] for the magnetic fieldinduced anisotropy using the effective action in the Schwinger proper-time formulation. The resulting anisotropic gauge theory can be simulated directly on the lattice. The setup and the simulation algorithm are described in appendix B. The main difference to simple pure gauge theory amounts to multiplying the plaquettes lying in the $z-t$ plane by the anisotropy coefficient $\kappa$. The exact form of the anisotropic action is given in eq. (B.2).

Before discussing the lattice simulations of the anisotropic theory, let us make one more remark. Besides writing down the effective Lagrangian (4.1), ref. [43, 44] also predicted that the scale $\lambda_{\mathrm{QCD}}$ of this theory (generated through dimensional transmutation) is much smaller than the QCD scale at $B=0: \lambda_{\mathrm{QCD}} \ll \Lambda_{\mathrm{QCD}}$ for a very broad range of magnetic fields. In the absence of further dimensionful scales in the anisotropic theory, this implies that the deconfinement transition temperature for strong mangetic fields is also much smaller than $T_{c}(B=0)$. Below we will also address this prediction.

Due to the exact $\mathbb{Z}(3)$ symmetry of the anisotropic theory, the deconfinement transition is characterized by the projected Polyakov loop (2.6). In the left panel of figure 6, this observable is plotted against the inverse gauge coupling $\beta$ for several values of $\kappa$, as measured on the $16^{3} \times 4$ lattices. At $\kappa=0$, we reproduce the results of refs. $[49,50]-$ in particular, the deconfinement transition occurs at $\beta_{c} \approx 4.07$. The results indicate $\beta_{c}$ to be strongly reduced as $\kappa$ grows. ${ }^{1}$ We find that $\beta_{c}$ scales approximately with $1 / \sqrt{\kappa}$, see the right panel of figure 6 . Extrapolating to $\kappa=\infty$ we obtain $\beta_{c}(\kappa=\infty) \approx 2.42(5)$. Besides approaching this limit via finite values of the anisotropy coefficient, we also develop an algorithm to simulate directly at $\kappa=\infty$. The corresponding setup is described in appendix B.

The left panel of figure 7 shows $P^{\text {pr }}$ at infinite anisotropy. We find that the critical inverse coupling on the $16^{3} \times 4$ lattice is comparable with the extrapolation based on

\footnotetext{
${ }^{1}$ Here we simulated at fixed values of the ratio $\kappa / \beta$. This continuous rescaling of $\kappa$ has no effect on, for example, the critical inverse coupling.
} 

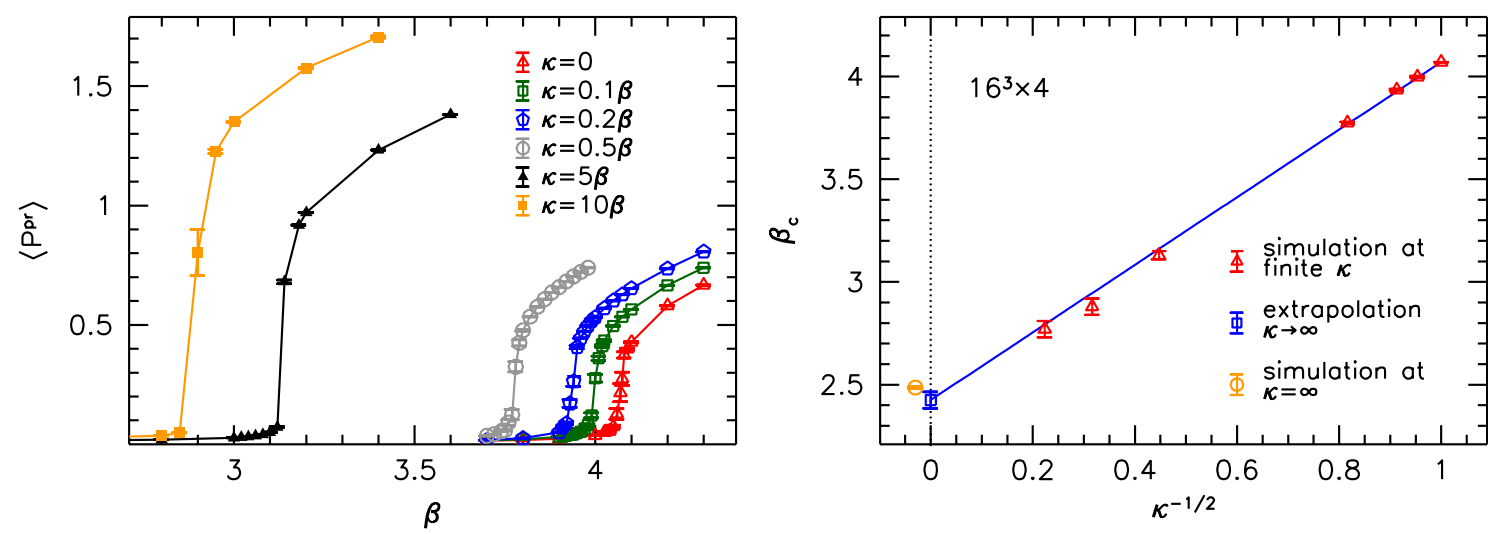

Figure 6. Left panel: the projected Polyakov loop as a function of the inverse gauge coupling for various values of the anisotropy coefficient, as measured on the $16^{3} \times 4$ lattices. The solid lines merely serve to guide the eye. Right panel: the critical inverse coupling as a function of $1 / \sqrt{\kappa}$. The extrapolation to $\kappa=\infty$ is compared to the result of the direct simulation at infinite anisotropy (the latter shifted horizontally for better visibility).
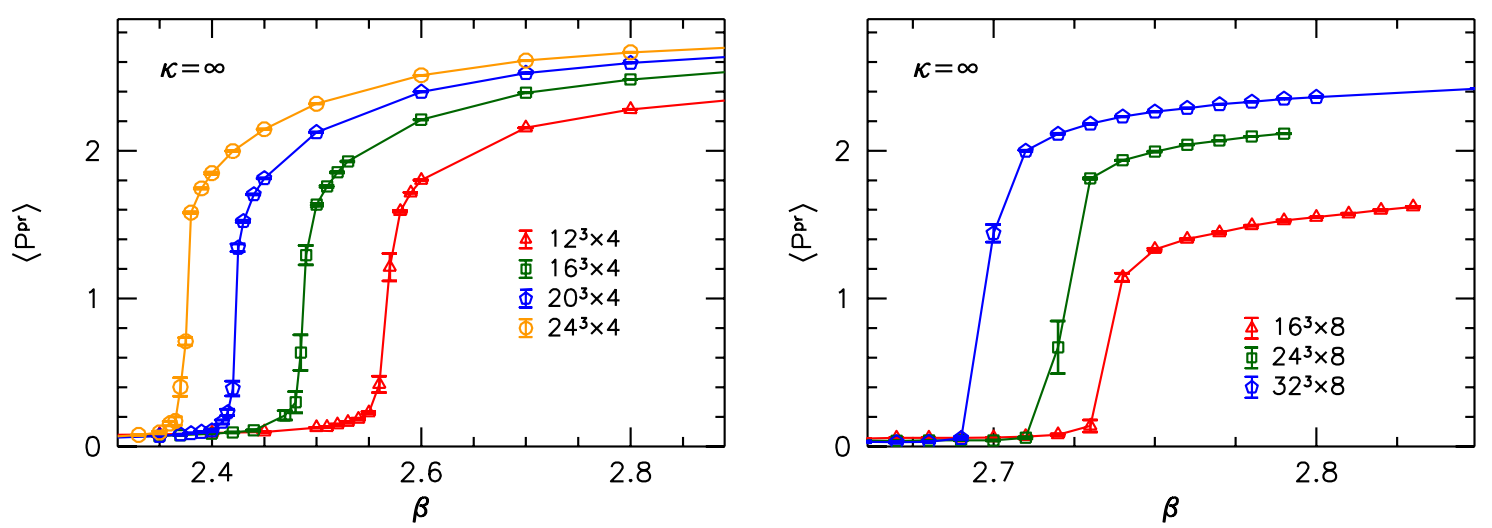

Figure 7. The projected Polyakov loop as a function of the inverse gauge coupling at $\kappa=\infty$, for various lattice volumes with $N_{t}=4$ (left panel) and $N_{t}=8$ (right panel). The solid lines merely serve to guide the eye.

finite anisotropies, see the right panel of figure 6 . In addition, a comparison of the results at different spatial volumes $12^{3} \ldots 24^{3}$ reveals that the transition becomes sharper as the volume increases, as typical for real phase transitions. We also repeated this analysis on $N_{t}=8$ lattices, see the right panel of figure 7 . The critical couplings are clearly different, showing that the transition is indeed related to the finite temperature. We also mention that finite volume effects in $\beta_{c}$ are observed to be unusually large - above $10 \%$ for $N_{t}=4$. (For comparison, the finite volume effects at $\kappa=0$ on similar lattices are of $0.1 \%$ [49].) We suspect that this is due to lattice artefacts - indeed, the effect is considerably smaller for $N_{t}=8$, see the right panel of figure 7 .

To determine the nature of the transition, we calculated the susceptibility of the pro- 

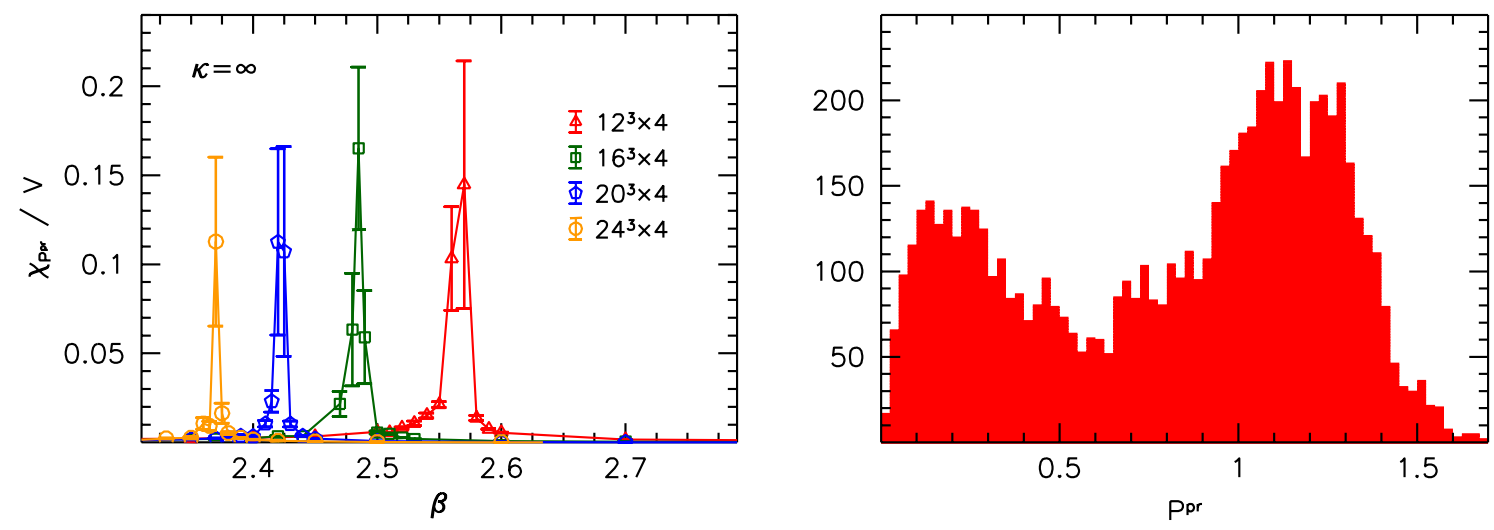

Figure 8. Left panel: the susceptibility of the projected Polyakov loop, normalized by the spatial volume, as a function of the inverse coupling. Various spatial volumes with $N_{t}=4$ are compared. The solid lines serve to guide the eye. Right panel: histogram of $P^{p r}$ near the critical temperature on the $16^{3} \times 4$ lattices.

jected Polyakov loop (2.7). This observable is shown in the left panel of figure 8 , with a normalization by the spatial volume. Within statistical errors, the height of the normalized susceptibility peak is observed to be independent of $V$. In other words, the peak height scales linearly with $V$, which we take as strong evidence that the transition is of first order. The histogram of $P^{\mathrm{pr}}$ at $\beta=2.4855$ as measured on the $16^{3} \times 4$ lattices is shown in the right panel of figure 8 , revealing the two-peak structure characteristic for first-order transitions.

Through the equivalence between this anisotropic gauge theory and QCD with asymptotically strong magnetic fields, the above finding implies that the QCD phase diagram exhibits a critical point in the strong magnetic field region, where the crossover turns into a real phase transition. Based on our full QCD results for the light quark susceptibilities, we will estimate the magnetic field corresponding to the critical point in section 5 .

The next step is to relate the critical inverse coupling $\beta_{c}$ to the critical temperature $T_{c}$ in physical units. To do so, we must set the lattice scale $\beta(a)$. In principle, the magnetic field is not expected to change this scaling relation (cf. ref. [6]). However, to arrive at our anisotropic gauge theory, $B$ has been taken to infinity, i.e. it also exceeds the squared lattice cutoff $a^{-2}$. Clearly, the lattice scale determined at $B=0$ becomes invalid beyond this point. Thus, in order to determine the lattice spacing, one needs a dimensionful quantity whose value is known in the asymptotic limit - for example a purely gluonic observable, where the $B$-dependence is expected to be only mild. A possible candidate for this role is the parameter $w_{0}$ defined from the gradient flow of the gauge links [51] that is often used for scale setting in QCD, as suggested in ref. [52].

We determined $w_{0}$ on our zero-temperature full QCD ensembles [7] for $e B<1 \mathrm{GeV}^{2}$ and also for $e B=3.25 \mathrm{GeV}^{2}$ at our lowest temperature $T \approx 75 \mathrm{MeV}$. The results for the ratio $w_{0} / w_{0}(B=0)$ are plotted in the left panel of figure 9 , showing a mild reduction of this parameter as $B$ grows. A fit of the form similar to eq. (3.1) describes the data well and suggests a saturation towards the asymptotically strong magnetic field limit. Nevertheless, 

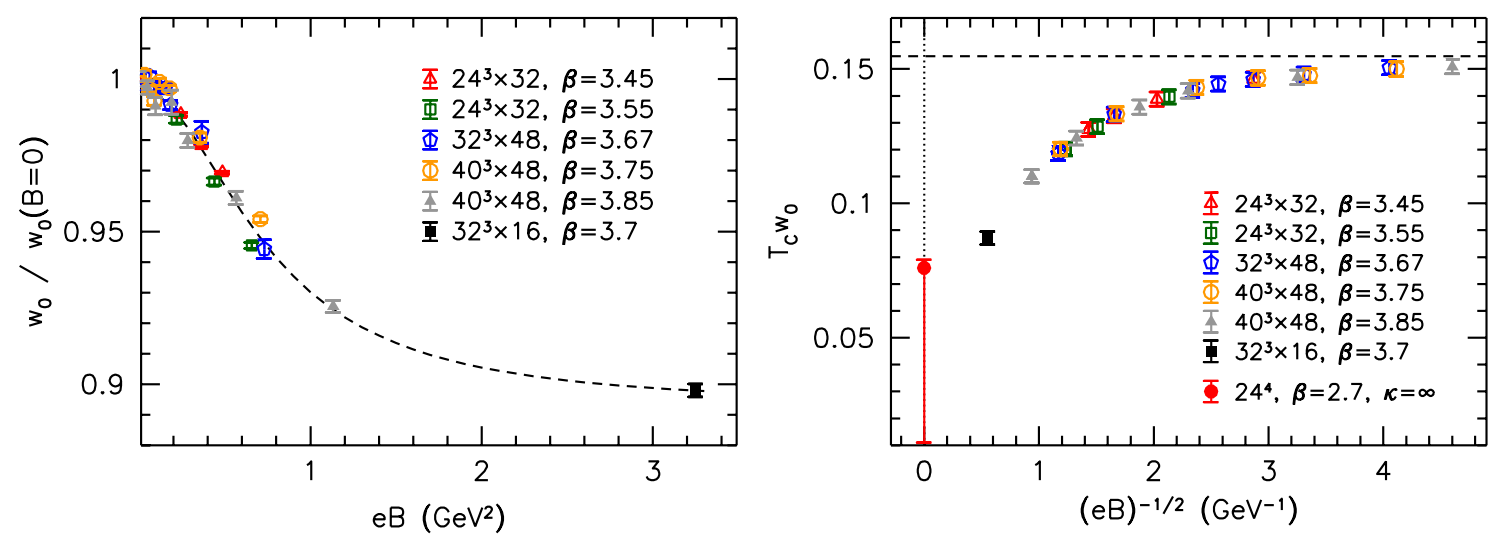

Figure 9. Left panel: magnetic field-dependence of the parameter $w_{0}$ using various lattice spacings and a fit (dashed line) of the form similar to eq. (3.1). Right panel: the dimensionless combination $T_{c} w_{0}$ in full QCD $\left(1 / \sqrt{e B}>0.5 \mathrm{GeV}^{-1}\right)$ and in the anisotropic pure gauge theory $(1 / \sqrt{e B}=0)$. The dashed line indicates the $B=0$ limit.

we cannot exclude a significant dependence of $w_{0}$ on $B$ for $B>3.25 \mathrm{GeV}^{2}$.

In addition, we can also gain some insight by considering the dimensionless combination $T_{c} w_{0}$. How close full $\mathrm{QCD}$ at $e B=3.25 \mathrm{GeV}^{2}$ is to the asymptotic limit can then be quantified by matching $T_{c} w_{0}$ with the anisotropic theory. Multiplying our full QCD results for $w_{0}$ by the transition temperature (here we take the definition of $T_{c}$ employing the inflection point of the strange quark number susceptibility, cf. figure 5$), T_{c} w_{0}$ is shown in the right panel of figure 9. Motivated by the scaling of $\beta_{c}$ (cf. the right panel of figure 6), the results are plotted against $1 / \sqrt{e B}$. Employing the result for $w_{0}$ from ref. [52], at zero magnetic field we have $T_{c}(B=0) \cdot w_{0}(B=0)=0.174(3) \mathrm{GeV} \cdot 0.1755(19) \mathrm{fm}=0.155(3)$.

To carry out the comparison to the asymptotic limit, we also determined $w_{0} / a$ on symmetric $16^{4}$ and $24^{4}$ anisotropic gauge configurations at the critical couplings corresponding to the $16^{3} \times 4\left(\beta_{c} \approx 2.47\right)$ and to the $24^{3} \times 8\left(\beta_{c} \approx 2.7\right)$ lattices. $^{2}$ We observe that the combination $T_{c} w_{0}=w_{0}\left(\beta_{c}\right) / a \cdot 1 / N_{t}$ - similarly to $\beta_{c}$ - suffers from large lattice discretization effects and exhibits a downwards trend towards the continuum limit. We take the result for the $N_{t}=8$ data as an upper limit, giving $\lim _{B \rightarrow \infty}\left(T_{c} w_{0}\right) \lesssim 0.076$. This value is also included in the right panel of figure 9. Altogether, the results are compatible with a monotonous dependence of $T_{c} w_{0}$ on $B$. To extrapolate $T_{c} w_{0}$ reliably to the continuum limit in the anisotropic theory requires further simulations on finer lattices and will be discussed in a forthcoming publication.

To summarize, the lattice results favor a saturation of $w_{0}$ and a monotonous reduction of $T_{c} w_{0}$ as the limit $B \rightarrow \infty$ is approached. This suggests a monotonous reduction of $T_{c}(B)$ towards the asymptotic limit. Nevertheless, based on the available findings, no final statement about $\lim _{B \rightarrow \infty} T_{c}$ can be made.

Let us make one more remark about the $\kappa=\infty$ anisotropic theory. Since the parallel chromoelectric component $\operatorname{tr} \mathcal{E}_{\|}^{2}$ of the action vanishes, all plaquettes lying in the $z-t$ plane

\footnotetext{
${ }^{2}$ Just as in full QCD, the gauge links are evolved here using the symmetric gradient flow.
} 
are unity. This implies that all Wilson loops $W$ in this plane are trivial, and the static quark-antiquark potential $\propto \log W$ is independent of the distance. Accordingly, there is no force acting on quark-antiquark pairs if they are separated in the direction of the magnetic field, i.e. the string tension $\sigma_{\|}$in this direction vanishes. This is in line with recent lattice determinations of the string tension in magnetic fields [53].

\section{Conclusions}

In this paper, we determined the nature and the characteristic temperature of the chi$\mathrm{ral} /$ deconfinement transition of QCD at an extremely strong background magnetic field $e B=3.25 \mathrm{GeV}^{2}$. The results for various observables consistently show that the transition temperature is further decreased compared to its value at lower magnetic fields. For the light quark condensates, the reduction of $T_{c}$ is due to the so-called inverse magnetic catalysis: between $e B=1 \mathrm{GeV}^{2}$ and $e B=3.25 \mathrm{GeV}^{2}, \Sigma_{u}$ and of $\Sigma_{d}$ are significantly reduced in the transition region. At the same time, the condensates are enhanced by $B$ both for $T \ll T_{c}$ and for $T \gg T_{c}$ (the latter effect is small, since the condensate is suppressed at high temperatures).

Comparing the behavior of the up and down quark condensates and that of the Polyakov loop also revealed that there is no splitting between the transition temperatures for the individual flavors, neither is there significant difference between the chiral and the deconfinement transition temperatures. On the contrary, the different definitions of $T_{c}$ tend to approach each other as $B$ grows and at $e B=3.25 \mathrm{GeV}^{2}$ all observables exhibit a single transition temperature of around $109-112 \mathrm{MeV}$, see figure 5. Furthermore, we performed a finite size scaling analysis of the light quark susceptibilities, which has revealed that there is no singularity in the infinite volume limit and, thus, the transition remains an analytic crossover even at $e B=3.25 \mathrm{GeV}^{2}$.

In addition, we considered the asymptotically strong magnetic field limit, and simulated the corresponding effective theory on the lattice. This limiting effective theory - an anisotropic pure gauge theory — was found to exhibit a first-order deconfinement phase transition. Together with our findings above, this implies the existence of a critical point in the QCD phase diagram. To provide a first estimate for the magnetic field $B_{\mathrm{CP}}$ corresponding to the critical point, let us return to our results about the width $w(B)$ of the light quark susceptibilities. We have seen that the width is reduced as the magnetic field grows, see the right panel of figure 4. Assuming a linear dependence of $w$ on $B$ and extrapolating in the magnetic field we find that $w$ vanishes at

$$
e B_{\mathrm{CP}} \approx 10(2) \mathrm{GeV}^{2}
$$

In light of the fact that the $B$-dependence of some of our observables (e.g. of $T_{c}$ and of $w_{0}$ ) tends to flatten out as $B$ grows, this first estimate should rather be taken as a lower bound for $B_{\mathrm{CP}}$. We mention that in order to simulate with magnetic fields of strengths comparable to that in eq. (5.1), lattices with $N_{t} \gtrsim 28$ are required, out of reach for current computational resources. 


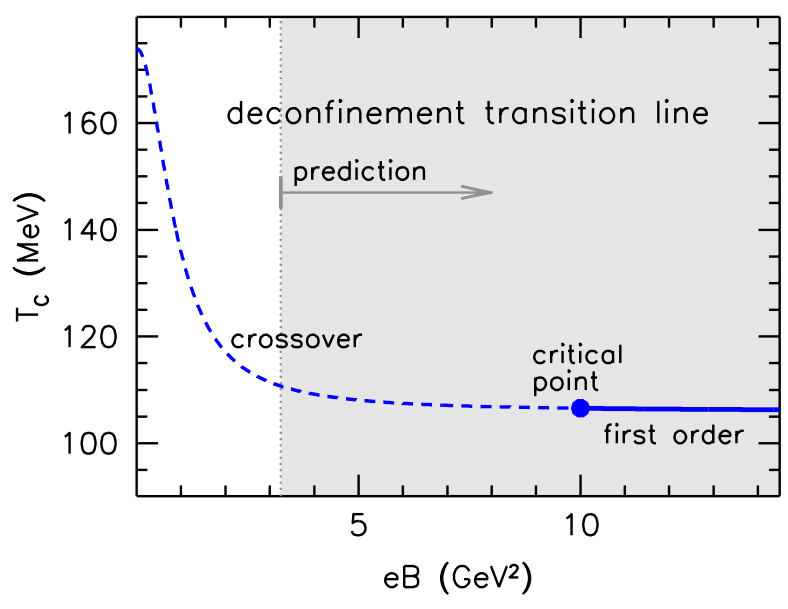

Figure 10. The deconfinement transition temperature against the background magnetic field. The results of our full lattice QCD simulations (white background) are complemented by the prediction (gray background) based on the results corresponding to the $B \rightarrow \infty$ limit and on the extrapolation of the light quark susceptibility peak to high magnetic fields (see the text).

In the absence of a priori known dimensionful scales in the $B \rightarrow \infty$ system, we could not determine $\lim _{B \rightarrow \infty} T_{c}$ in physical units. Nevertheless, the deconfinement transition temperature of the anisotropic theory is expected to be much smaller than $T_{c}(B=0)[43$, 44]. ${ }^{3}$ Our results for the combination $T_{c} w_{0}$ are compatible with this prediction and suggest a gradual reduction of the deconfinement transition temperature as $B$ is increased. ${ }^{4}$ Taking these aspects into account, figure 10 represents a sketch of the deconfinement transition line in the QCD phase diagram for a broad range of magnetic fields.

The reader might wonder whether it is possible that the crossover at $e B \leq 3.25 \mathrm{GeV}^{2}$ and the first-order transition in the asymptotic limit are not connected by a single line. To see that this is not the case, note that by varying the anisotropy parameter $\kappa$, one can continuously deform the anisotropic theory to usual pure gauge theory, as was demonstrated in figure 6. Furthermore, the isotropic pure gauge theory can be thought of as QCD with infinitely heavy quarks and thus can be continuously transformed into full QCD by increasing the inverse quark masses from zero to their physical values. Thus, the transition we identified at $B \rightarrow \infty$ is indeed the same deconfinement transition that occurs at low magnetic fields.

\footnotetext{
${ }^{3}$ The discussion in ref. [43, 44] bases on renormalization group arguments and on the separation of scales $\lambda_{\mathrm{QCD}} \ll m_{d} \ll \sqrt{e B}$ (here $\lambda_{\mathrm{QCD}}$ is the dynamical scale of the large- $B$ theory) to conclude that $\lambda_{\mathrm{QCD}} \ll \Lambda_{\mathrm{QCD}}$ for a very broad range of magnetic fields - in fact, up to $e B$ being millions of orders of magnitudes larger than $\Lambda_{Q C D}^{2}$. To find the complete $B$-dependence of the running of the strong coupling and to prove rigorously that $\lambda_{\mathrm{QCD}} \ll \Lambda_{\mathrm{QCD}}$ even in the $B \rightarrow \infty$ limit, a full treatment of the divergent one-loop Feynman diagrams in the presence of background magnetic fields would be necessary. Without relying on the lowest-Landau-level approximation — which might not be justified for the case of divergent diagrams - this is a very difficult task.

${ }^{4}$ Note that our setup at $B \rightarrow \infty$ describes the low-energy effective action for gluons. Thus, the results in the anisotropic theory have no implications for the chiral transition. For more details on this point, see ref. $[43,44]$.
} 
Let us highlight that according to this discussion, having a decreasing deconfinement transition temperature is actually natural to QCD. Furthermore, since the $B \rightarrow \infty$ limit is independent of the quark masses, ${ }^{5}$ a similar reduction of $T_{c}$ by the magnetic field should also take place in QCD with heavier-than-physical quarks. However, in the latter case this reduction most probably follows an initial increase in the transition temperature, cf. refs. [6, 54]. Indeed, recent lattice results employing overlap fermions and pion masses of about $500 \mathrm{MeV}$ indicate inverse catalysis to occur around the transition temperature at the magnetic field $e B \approx 1.3 \mathrm{GeV}^{2}[9]$.

Finally, we note that magnetic fields well above the strength (5.1) are predicted to be generated during the electroweak phase transition in the early universe [55]. If these fields remain strong enough until the QCD epoch, the emerging first-order phase transition might have several exciting consequences. Via supercooling, bubbles of the confined phase can be formed as the temperature drops below $T_{c}$, leading to large inhomogeneities, important for nucleosynthesis [56]. Collisions between the bubbles can also lead to the emission of gravitational waves and, thus, leave an imprint on the primordial gravitational spectrum [57]. An absence of such signals, in turn, would imply an upper limit for the strength of the primordial magnetic fields.

\section{Acknowledgments}

This work was supported by the DFG (SFB/TRR 55). The author thanks Igor Shovkovy for valuable comments and Gunnar Bali, Bastian Brandt, Falk Bruckmann, Jan Pawlowski, Kálmán Szabó and Andreas Schäfer for enlightening discussions.

\section{A Effective action in the asymptotic magnetic field limit}

In this appendix we demonstrate how asymptotically strong magnetic fields induce an anisotropy in the gluonic sector using an Euler-Heisenberg-type approach. The QCD effective Lagrangian in Euclidean space-time is

$$
\mathcal{L}(B)=\frac{1}{2 g^{2}} \operatorname{tr} G_{\mu \nu} G_{\mu \nu}+\mathcal{L}^{q}\left(B, G_{\mu \nu}\right), \quad \mathcal{L}^{q}\left(B, G_{\mu \nu}\right)=-\sum_{f=u, d, s} \log \operatorname{det}\left[\not D\left(q_{f} B, G_{\mu \nu}\right)+m_{f}\right],
$$

and the quark determinant will be regularized using Schwinger's proper time formulation [58]. Since the electromagnetic field exceeds all scales in the system and in particular, $(e B)^{2} \gg \operatorname{tr} G_{\mu \nu}^{2}$, we may approximate the the chromo-fields in the fermionic action to be weak. In addition, we assume the chromo-fields to be covariantly constant, $D_{\mu} G_{\nu \rho}=0$ to enable a fully analytical treatment of the problem. Given this condition, the field strength can be gauge transformed to be constant in space-time and diagonal in color space [59], $G_{\mu \nu}=\operatorname{diag}\left(G_{\mu \nu c}\right)$ with the color index $c=1,2,3$.

\footnotetext{
${ }^{5}$ As long as the quark masses are finite - note that the $m \rightarrow \infty$ and $B \rightarrow \infty$ limits cannot be interchanged.
} 
Let us decompose the chromo-fields to chromomagnetic/chromoelectric components,

$$
\mathcal{B}_{\|}=G_{x y}, \quad \mathcal{B}_{\perp}=\frac{G_{x z}+G_{y z}}{2}, \quad \mathcal{E}_{\|}=G_{z t}, \quad \mathcal{E}_{\perp}=\frac{G_{x t}+G_{y t}}{2},
$$

parallel or perpendicular to the electromagnetic field $B$. The leading terms in the strong $B$-expansion are quadratic in the chromo-fields and thus, to find the coefficients of the respective components, it suffices to consider separately the effect of $B$ and $\mathcal{B}_{\|}, B$ and $\mathcal{B}_{\perp}, B$ and $\mathcal{E}_{\|}$and $B$ and $\mathcal{E}_{\perp}$. The effective Lagrangian for these components for small background magnetic fields was determined in refs. [48, 60]. A similar calculation, generalized to finite temperatures and constant Polyakov loop backgrounds was performed in refs. [8, 61].

Let us first take the case of $B$ and $\mathcal{B}_{\|}$. For each flavor we may choose our coordinate system such that $q_{f} B$ is positive. Then, each color component experiences a total (positive) magnetic field $q_{f} B+\mathcal{B}_{\| c}$ so that

$$
\mathcal{L}^{q}\left(B, \mathcal{B}_{\|}\right)=\frac{1}{8 \pi^{2}} \sum_{f, c} m_{f}^{2}\left(q_{f} B+\mathcal{B}_{\| c}\right) \int \frac{\mathrm{d} s}{s^{2}} e^{-s} \operatorname{coth} \frac{\left(q_{f} B+\mathcal{B}_{\| c}\right) s}{m_{f}^{2}} .
$$

Since $\mathcal{B}_{\| c}$ only appears in the sum with $q_{f} B$, the effective Lagrangian becomes independent of the chromomagnetic field in the limit $q_{f} B \gg \mathcal{B}_{\| c}$. This implies that quarks become insensitive to $\mathcal{B}_{\|}$, i.e. decouple from this gluonic component.

Next we take the case with $B$ and $\mathcal{B}_{\perp}$. Rotating our coordinate axes for each color component such that the $z$ axis points in the direction of the total magnetic field we get

$$
\mathcal{L}^{q}\left(B, \mathcal{B}_{\perp}\right)=\frac{1}{8 \pi^{2}} \sum_{f, c} m_{f}^{2} \sqrt{\left(q_{f} B\right)^{2}+\mathcal{B}_{\perp c}^{2}} \int \frac{\mathrm{d} s}{s^{2}} e^{-s} \operatorname{coth} \frac{\sqrt{\left(q_{f} B\right)^{2}+\mathcal{B}_{\perp c}^{2}} s}{m_{f}^{2}}
$$

In the strong $B$ limit, this becomes independent of $\mathcal{B}_{\perp c}$, signaling that quarks decouple from the perpendicular chromomagnetic component of the gluons as well.

For a perpendicular chromoelectric field, for each color component we can perform the Lorentz transformation that eliminates the electric field and, in turn, gives a total magnetic field $\sqrt{\left(q_{f} B\right)^{2}+\mathcal{E}_{\perp c}^{2}}$. The corresponding effective Lagrangian equals eq. (A.4), but with $\mathcal{B}_{\perp c}$ replaced by $\mathcal{E}_{\perp c}$. This implies the decoupling of quarks from the perpendicular chromoelectric fields.

Finally, for a parallel chromoelectric field $\mathcal{E}_{\|}$, we have a Landau problem in the $x-y$ as well as in the $z-t$ planes, giving

$$
\mathcal{L}^{q}\left(B, \mathcal{E}_{\|}\right)=\frac{1}{8 \pi^{2}} \sum_{f, c} q_{f} B \mathcal{E}_{\| c} \int \frac{\mathrm{d} s}{s} e^{-s} \operatorname{coth} \frac{q_{f} B s}{m_{f}^{2}} \operatorname{coth} \frac{\mathcal{E}_{\| c} s}{m_{f}^{2}} .
$$

Taking the limit $q_{f} B \gg \mathcal{E}_{\| c}, m_{f}^{2}$, we see that - unlike for the other components above - a non-trivial dependence on $\mathcal{E}_{\|}$remains. We are interested in the quadratic term, 
proportional to $\operatorname{tr} \mathcal{E}_{\|}^{2}$, which contributes ${ }^{6}$ to the gluonic Lagrangian $\operatorname{tr} G_{\mu \nu}^{2}$ of eq. (A.1),

$$
\mathcal{L}^{q}\left(B, \mathcal{O}\left(\mathcal{E}_{\|}^{2}\right)\right)=\frac{1}{24 \pi^{2}} \sum_{f, c} q_{f} B \frac{\mathcal{E}_{\| c}^{2}}{m_{f}^{2}} \int \mathrm{d} s e^{-s}=\kappa(B) \operatorname{tr} \mathcal{E}_{\|}^{2}, \quad \kappa(B) \equiv \frac{1}{24 \pi^{2}} \sum_{f}\left|q_{f} / e\right| \frac{|e B|}{m_{f}^{2}} .
$$

Altogether, the asymptotically strong magnetic field limit of the QCD effective Lagrangian indeed equals eq. (4.1). Thus we find that the chromo-dielectric constant is enhanced in the direction of the background magnetic field, and the coefficient $\kappa(B)$ coincides with the result of ref. [43, 44].

Having $\kappa \gg 1$ in the action implies that the corresponding gluonic field strength component $\operatorname{tr} \mathcal{E}_{\|}^{2}$ is strongly suppressed. In other words, the anisotropy in the chromoelectric part of the action density,

$$
A(\mathcal{E})=\frac{1}{V_{4}} \frac{1}{g^{2}}\left\langle\operatorname{tr} \mathcal{E}_{\perp}^{2}-\operatorname{tr} \mathcal{E}_{\|}^{2}\right\rangle
$$

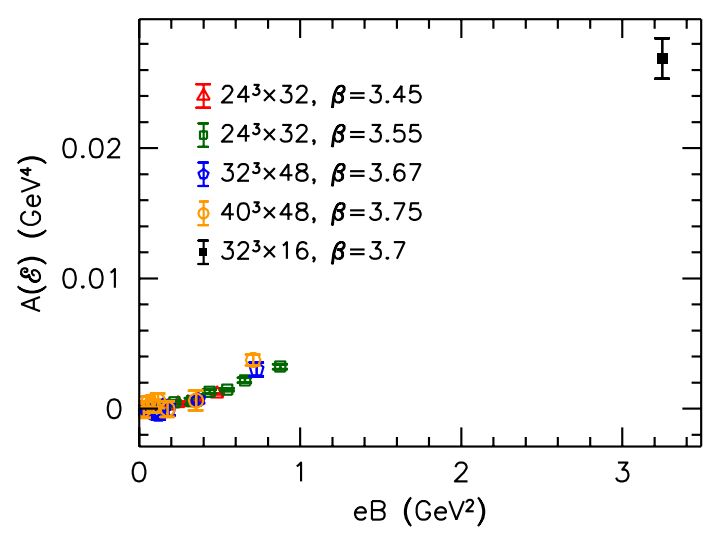

is enhanced by $B$. To back up this prediction, in figure 11 we plot $A(\mathcal{E})$ as a function of the magnetic field, based on our zerotemperature results at $e B<1 \mathrm{GeV}^{2}[48]$ and the measurements at $e B=3.25 \mathrm{GeV}^{2}$ at our lowest temperature $T \approx 75 \mathrm{MeV}$. The results clearly indicate that the anisotropy is positive and strongly increased as $B$ grows. We note that due to the coupling between the gluonic field strength components, a similar anisotropy in the chromomagnetic sector also appears, altogether giving rise to the hierarchy $\operatorname{tr} \mathcal{B}_{\|}^{2}>\operatorname{tr} \mathcal{B}_{\perp}^{2}=\operatorname{tr} \mathcal{E}_{\perp}^{2}>\operatorname{tr} \mathcal{E}_{\|}^{2}$ at low temperatures. The same hierarchy is also observed in the anisotropic gauge theory. ${ }^{7}$

We note that the calculation leading to eq. (4.1) can also be performed for nonzero temperatures. At $T>0$ an additional factor appears in the proper time integral due to the sum over Matsubara frequencies, containing an elliptic $\Theta$-function. This factor decouples

\footnotetext{
${ }^{6}$ Here we omitted a divergent term of the form $\operatorname{tr} \mathcal{E}_{\|}^{2} \log \Lambda / m_{f}$, where $\Lambda$ is a cutoff entering as the lower endpoint of the proper time integration $s_{0} \propto 1 / \Lambda^{2}$. This divergence can be eliminated by the multiplicative renormalization of the wave function $\mathcal{E}_{\|}$and of the gauge coupling $g$ [58]. Closer inspection of eqs. (A.3) and (A.4) shows that the same type of divergence is present for the other components as well. Thus, these $B$-independent terms merely represent an isotropic redefinition of the gauge coupling $g$, which does not alter the form of the effective Lagrangian for strong magnetic fields. Another divergence, independent of the gluonic field strengths, takes the form $\left(q_{f} B\right)^{2} \log \Lambda / m_{f}$ and is canceled by the renormalization of $B$ and of $q_{f}$ [58]. Thus, the necessary renormalizations at $B \rightarrow \infty$ are of the same type as for the theory at small magnetic fields.

${ }^{7}$ To see how the anisotropic dielectric constant affects the chromomagnetic components, it is instructive to consider the gauge potential $A_{\mu}$. A large value of $\kappa$ implies a suppression of $\operatorname{tr} \mathcal{E}_{\|}^{2}$ and a corresponding suppression of the fluctuations in $A_{z}$ and in $A_{t}$. This suppression propagates into the magnetic sector and creates the anisotropy between $\operatorname{tr} \mathcal{B}_{\perp}^{2}$ and $\operatorname{tr} \mathcal{B}_{\|}^{2}$. Indeed, while the former contains $A_{z}$, the latter does not.
} 
from the $B$-dependence, implying that even for $T>0$, only the chromo-dielectric constant is affected. The coefficient $\kappa$ is, however, altered as

$$
\kappa(B, T)=\frac{1}{24 \pi^{2}} \sum_{f}\left|q_{f} / e\right| \frac{|e B|}{m_{f}^{2}} \int \mathrm{d} s e^{-s} \Theta_{3}\left[\frac{\pi}{2}, e^{-m_{f}^{2} /\left(4 s T^{2}\right)}\right] .
$$

The integral over $s$ equals unity at $T=0$ and is reduced monotonously (and smoothly) as the temperature grows. Simulating the anisotropic gauge theory according to the Lagrangian (4.1) on the lattice, we found that the theory exhibits a first-order phase transition. Thus, since the smooth $\kappa(T)$ dependence does not affect the discontinuous transition, in order to locate the critical temperature it suffices to simulate the theory at fixed (large) $\kappa$ values.

\section{B Simulating anisotropic pure gauge theory on the lattice}

In this appendix we discuss the simulation algorithm for the anisotropic pure gauge theory described by the Lagrangian (4.1). The corresponding path integral

$$
\mathcal{Z}=\int \mathcal{D} U e^{-\beta S_{g}^{\text {aniso }}}
$$

can be simulated directly on the lattice. Here, $U$ denotes the gauge links, $\beta=6 / g^{2}$ is the inverse gauge coupling and the anisotropic gauge action reads

$$
S_{g}^{\text {aniso }}=\sum_{\mu<\nu} \frac{1}{3} \operatorname{Re} \operatorname{tr} P_{\mu \nu} \cdot \kappa_{\mu \nu}, \quad \kappa_{\mu \nu}= \begin{cases}1+\kappa(B) / \beta, & \mu=z, \nu=t, \\ 1, & \text { otherwise }\end{cases}
$$

where $P_{\mu \nu}$ are linear combinations of closed loops lying in the $\mu-\nu$ plane. We take the treelevel Symanzik improved gauge action such that these loops include the $1 \times 1$ plaquettes $U_{\mu \nu}^{1 \times 1}$ and the $2 \times 1$ rectangles $U_{\mu \nu}^{2 \times 1}$ with appropriately tuned coefficients [62],

$$
P_{\mu \nu}=-\frac{1}{12}\left(\mathbb{1}-U_{\mu \nu}^{2 \times 1}\right)+\frac{5}{3}\left(\mathbb{1}-U_{\mu \nu}^{1 \times 1}\right) .
$$

The correspondence between the continuum and lattice expressions reads

$\operatorname{tr} \mathcal{E}_{\|}^{2}=2 \operatorname{Retr} P_{t z}, \quad \operatorname{tr} \mathcal{E}_{\perp}^{2}=\operatorname{Retr}\left[P_{t x}+P_{t y}\right], \quad \operatorname{tr} \mathcal{B}_{\|}^{2}=2 \operatorname{Retr} P_{x y}, \quad \operatorname{tr} \mathcal{B}_{\perp}^{2}=\operatorname{Retr}\left[P_{y z}+P_{x z}\right]$.

To simulate this theory, we use an overrelaxation/heatbath algorithm, based on the isotropic pure gauge implementation by the MILC collaboration [63]. One trajectory consists of one overrelaxation step followed by four heatbath steps. The simulation at finite anisotropy coefficient $\kappa$ simply involves multiplying the plaquettes and rectangles lying in the $z-t$ plane by $\kappa$. We observe that autocorrelation times grow large as $\kappa$ increases, similarly to the issue of critical slowing down of the isotropic theory at large $\beta$. This prohibits approaching $\kappa \rightarrow \infty$, necessary for the asymptotically strong magnetic field limit. However, it is possible to modify the algorithm to simulate directly at $\kappa=\infty$. In this limit, the $z-t$ component of the action and the remaining five components decouple, and 
the links are restricted to the subspace $\Omega[U]$ of configurations, where $P_{z t}$ is minimal. This subspace is defined by

$$
\Omega[U]=\left\{U_{\mu} \mid U_{z t}^{1 \times 1}=\mathbb{1}\right\} .
$$

Indeed, any fluctuation in the link variables that leads off of this subspace makes the action infinitely large and is thus forbidden. (Note that if $U_{z t}^{1 \times 1}$ equals the unit matrix, then so does $U_{z t}^{2 \times 1}$.)

We thus have to parameterize the subspace $\Omega[U]$ in terms of the gauge links $U_{\mu}$. Let us label the lattice sites by $n=\left(n_{x}, n_{y}, n_{z}, n_{t}\right)$ with $0 \leq n_{\mu}<N_{\mu}$. To find the parameterization of eq. (B.5), it is advantageous to fix the links to $\mathbb{1}$ on a so-called maximal tree. The specific choice for the tree is shown in the left panel of figure 12. (Note that FaddeevPopov fields are absent for such a gauge fixing [64].) In order to have unit plaquettes for $n_{z}<N_{z}-1$ and $n_{t}<N_{t}-1$, all $t$-links must be set to unity at these sites. To have unit plaquettes on the last $z$-slice, all $z$-links at $N_{z}-1$ must be set equal, denoted by $L_{z}$. Similarly, all the $t$-links at $N_{t}-1$ must be set equal, denoted by $L_{t}$, see the visualization in the right panel of figure 12. These remaining links correspond to the local Polyakov loops in the $z$ - and in the $t$-direction, lying in the $z-t$ plane at a given $n_{x}$ and $n_{y}$. Finally, to ensure that the plaquette at the corner $n_{z}=N_{z}-1, n_{t}=N_{t}-1$ is unity, we need $L_{z} L_{t} L_{z}^{\dagger} L_{t}^{\dagger}=\mathbb{1}$, i.e. $L_{z}$ and $L_{t}$ must commute. Altogether, the subspace in question reads

$$
\begin{aligned}
\Omega[U]=\left\{U_{\mu} \mid\right. & U_{z}(n)=\mathbb{1} \forall n_{z} \neq N_{z}-1, \\
& U_{t}(n)=\mathbb{1} \forall n_{t} \neq N_{t}-1, \\
& U_{z}\left(n_{x}, n_{y}, N_{z}-1, n_{t}\right)=L_{z}\left(n_{x}, n_{y}\right) \forall n_{t}, \\
& U_{t}\left(n_{x}, n_{y}, n_{z}, N_{t}-1\right)=L_{t}\left(n_{x}, n_{y}\right) \forall n_{z}, \\
& {\left.\left[L_{z}\left(n_{x}, n_{y}\right), L_{t}\left(n_{x}, n_{y}\right)\right]=0\right\} . }
\end{aligned}
$$

Notice that the 'degenerate' timelike Polyakov loop $L_{t}$ (represented by the blue arrows in the right panel of figure 12) appears multiple times in the action - in fact, in $4 N_{z}$ plaquettes and in $12 N_{z}$ rectangles. The corresponding 'staples' are all taken into account in the update of $L_{t}$ (and similarly for $L_{z}$ ).

There are several ways to fulfill the commutativity relation $\left[L_{z}, L_{t}\right]=0$. One possibility (setup A) is to simply set $L_{z}=\mathbb{1}$ for all $n_{x}$ and $n_{y}$. Another approach (setup B) is to constrain $L_{z}$ to be a center element, $L_{z}\left(n_{x}, n_{y}\right) \in \mathbb{Z}_{3}$. The two setups only differ on a set whose measure vanishes in the limit, where all lattice extents are taken to infinity. Note that the expectation value of the average $z$-Polyakov loop $P^{(z)}$ [defined similarly as the usual Polyakov loop $P$, eq. (2.5)] is three for setup A, whereas it is zero for setup B, if the spatial size of the system is large enough. Nevertheless, we checked that observables sensitive to the finite temperature transition $(P$, the gauge action, etc.) all have vanishing correlators with $P^{(z)}$. In fact, we found that the setups $\mathrm{A}$ and $\mathrm{B}$ give identical results for $P$ and for the gauge action for all values of the inverse gauge coupling $\beta$ on the $16^{3} \times 4$ lattices. In other words, center symmetry breaking in the $z$ direction appears to be completely irrelevant for the deconfinement phase transition.

In addition, we also tried allowing both $L_{z}$ and $L_{t}$ to be general $\mathrm{SU}(3)$ matrices (which violates the commutativity relation). This approach (setup C) turned out to introduce 

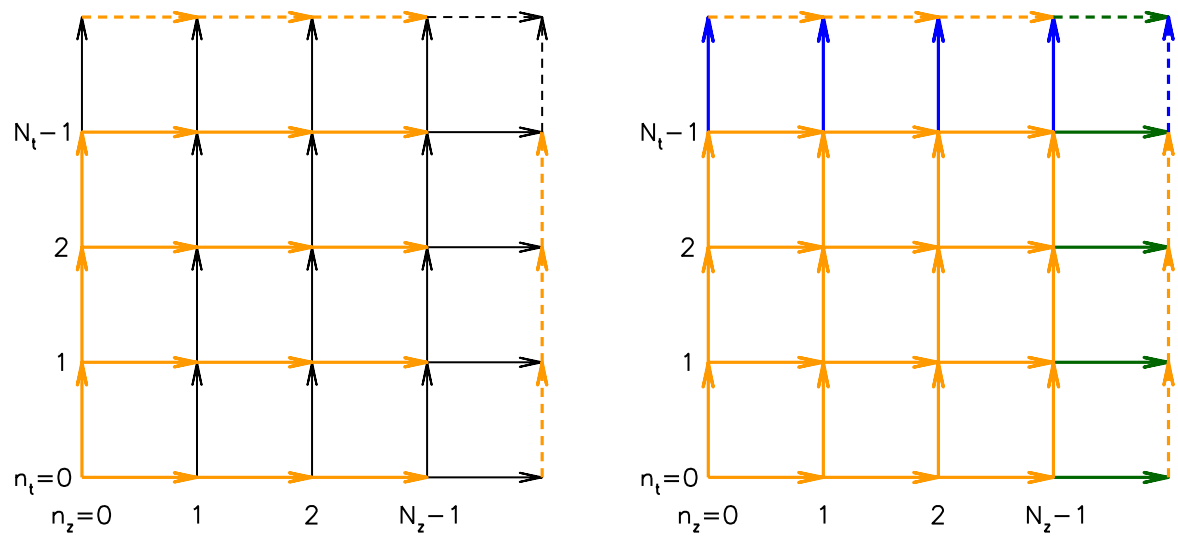

Figure 12. One $z-t$ plane of the lattice with periodic boundary conditions in both directions. The dashed arrows at $n_{z}=N_{z}\left(n_{t}=N_{t}\right)$ indicate the copies of the links at $n_{z}=0\left(n_{t}=0\right)$. Left panel: the gauge links on the maximal tree (yellow arrows) are fixed to the unit matrix. Right panel: in order for the configuration to belong to the subspace (B.6), further gauge links are set to unity (yellow) and the green and blue links are set equal, respectively. Finally, the blue and green 'degenerate' Polyakov loops must commute with each other.

negligible differences in the results. ${ }^{8}$ For the simulations presented in the body of the paper, we considered setup A and set $L_{z}=\mathbb{1}$ throughout the lattice. As a consistency check, besides the overrelaxation/heatbath algorithm, we also considered a hybrid MonteCarlo update and found that the two give fully consistent results.

Open Access. This article is distributed under the terms of the Creative Commons Attribution License (CC-BY 4.0), which permits any use, distribution and reproduction in any medium, provided the original author(s) and source are credited.

\section{References}

[1] Y. Aoki, G. Endrodi, Z. Fodor, S.D. Katz and K.K. Szabo, The Order of the quantum chromodynamics transition predicted by the standard model of particle physics, Nature $\mathbf{4 4 3}$ (2006) 675 [hep-lat/0611014] [INSPIRE].

[2] T. Bhattacharya et al., QCD Phase Transition with Chiral Quarks and Physical Quark Masses, Phys. Rev. Lett. 113 (2014) 082001 [arXiv:1402.5175] [INSPIRE].

[3] D. Kharzeev, K. Landsteiner, A. Schmitt and H.-U. Yee, Strongly Interacting Matter in Magnetic Fields, Lect.Notes Phys. 871 (2013) 1-624

[4] J.O. Andersen, W.R. Naylor and A. Tranberg, Phase diagram of QCD in a magnetic field: A review, arXiv:1411.7176 [INSPIRE].

[5] D.E. Kharzeev, Topology, magnetic field and strongly interacting matter, Ann. Rev. Nucl. Part. Sci. 65 (2015) 0000 [arXiv:1501.01336] [INSPIRE].

\footnotetext{
${ }^{8}$ The most general prescription is obtained by gauge transforming $L_{t}$ to diagonal form. The commuta-
} tivity relation then ensures that also $L_{z}$ is diagonal. In the infinite lattice size limit, this setup ought to give identical results as well. We leave this check for a future study. 
[6] G.S. Bali et al., The QCD phase diagram for external magnetic fields, JHEP 02 (2012) 044 [arXiv:1111.4956] [INSPIRE].

[7] G.S. Bali, F. Bruckmann, G. Endrodi, Z. Fodor, S.D. Katz and A. Schafer, QCD quark condensate in external magnetic fields, Phys. Rev. D 86 (2012) 071502 [arXiv:1206.4205] [INSPIRE].

[8] F. Bruckmann, G. Endrodi and T.G. Kovacs, Inverse magnetic catalysis and the Polyakov loop, JHEP 04 (2013) 112 [arXiv: 1303.3972] [INSPIRE].

[9] V.G. Bornyakov, P.V. Buividovich, N. Cundy, O.A. Kochetkov and A. Schäfer, Deconfinement transition in two-flavor lattice $Q C D$ with dynamical overlap fermions in an external magnetic field, Phys. Rev. D 90 (2014) 034501 [arXiv:1312.5628] [INSPIRE].

[10] V.P. Gusynin, V.A. Miransky and I.A. Shovkovy, Dimensional reduction and catalysis of dynamical symmetry breaking by a magnetic field, Nucl. Phys. B 462 (1996) 249 [hep-ph/9509320] [INSPIRE].

[11] S. Schramm, B. Müller and A.J. Schramm, Quark - anti-quark condensates in strong magnetic fields, Mod. Phys. Lett. A 7 (1992) 973 [INSPIRE].

[12] G. Endrödi, QCD equation of state at nonzero magnetic fields in the Hadron Resonance Gas model, JHEP 04 (2013) 023 [arXiv:1301.1307] [INSPIRE].

[13] G.S. Bali, F. Bruckmann, G. Endrd̈i, S.D. Katz and A. Schäfer, The QCD equation of state in background magnetic fields, JHEP 08 (2014) 177 [arXiv: 1406.0269] [INSPIRE].

[14] G. Endrödi, QCD in magnetic fields: from Hofstadter's butterfly to the phase diagram, PoS (LATTICE2014) 018 [arXiv: 1410.8028] [INSPIRE].

[15] E.S. Fraga, Thermal chiral and deconfining transitions in the presence of a magnetic background, Lect. Notes Phys. 871 (2013) 121 [arXiv:1208.0917] [INSPIRE].

[16] K. Fukushima and Y. Hidaka, Magnetic Catalysis Versus Magnetic Inhibition, Phys. Rev. Lett. 110 (2013) 031601 [arXiv: 1209.1319] [INSPIRE].

[17] J.O. Andersen and A.A. Cruz, Two-color QCD in a strong magnetic field: The role of the Polyakov loop, Phys. Rev. D 88 (2013) 025016 [arXiv:1211.7293] [INSPIRE].

[18] E.S. Fraga and L.F. Palhares, Deconfinement in the presence of a strong magnetic background: an exercise within the MIT bag model, Phys. Rev. D 86 (2012) 016008 [arXiv: 1201.5881] [INSPIRE].

[19] E.S. Fraga, J. Noronha and L.F. Palhares, Large- $N_{c}$ Deconfinement Transition in the Presence of a Magnetic Field, Phys. Rev. D 87 (2013) 114014 [arXiv:1207.7094] [INSPIRE].

[20] J. Chao, P. Chu and M. Huang, Inverse magnetic catalysis induced by sphalerons, Phys. Rev. D 88 (2013) 054009 [arXiv:1305.1100] [INSPIRE].

[21] K. Kamikado and T. Kanazawa, Chiral dynamics in a magnetic field from the functional renormalization group, JHEP 03 (2014) 009 [arXiv: 1312.3124] [INSPIRE].

[22] M. Ferreira, P. Costa, D.P. Menezes, C. Providência and N. Scoccola, Deconfinement and chiral restoration within the $\mathrm{SU}(3)$ Polyakov-Nambu-Jona-Lasinio and entangled Polyakov-Nambu-Jona-Lasinio models in an external magnetic field, Phys. Rev. D 89 (2014) 016002 [arXiv: 1305.4751 ] [INSPIRE].

[23] E.J. Ferrer, V. de la Incera, I. Portillo and M. Quiroz, New look at the QCD ground state in a magnetic field, Phys. Rev. D 89 (2014) 085034 [arXiv:1311.3400] [INSPIRE]. 
[24] E.J. Ferrer, V. de la Incera and X.J. Wen, Quark Antiscreening at Strong Magnetic Field and Inverse Magnetic Catalysis, Phys. Rev. D 91 (2015) 054006 [arXiv:1407.3503] [InSPIRE].

[25] S. Fayazbakhsh and N. Sadooghi, Anomalous magnetic moment of hot quarks, inverse magnetic catalysis and reentrance of the chiral symmetry broken phase, Phys. Rev. D 90 (2014) 105030 [arXiv:1408.5457] [INSPIRE].

[26] R.L.S. Farias, K.P. Gomes, G.I. Krein and M.B. Pinto, Importance of asymptotic freedom for the pseudocritical temperature in magnetized quark matter, Phys. Rev. C 90 (2014) 025203 [arXiv: 1404.3931] [INSPIRE].

[27] M. Ferreira, P. Costa, O. Lourenço, T. Frederico and C. Providência, Inverse magnetic catalysis in the (2+1)-flavor Nambu-Jona-Lasinio and Polyakov-Nambu-Jona-Lasinio models, Phys. Rev. D 89 (2014) 116011 [arXiv:1404.5577] [INSPIRE].

[28] A. Ayala, M. Loewe, A.J. Mizher and R. Zamora, Inverse magnetic catalysis for the chiral transition induced by thermo-magnetic effects on the coupling constant, Phys. Rev. D 90 (2014) 036001 [arXiv:1406.3885] [INSPIRE].

[29] A. Ayala, M. Loewe and R. Zamora, Inverse magnetic catalysis in the linear $\sigma$-model with quarks, Phys. Rev. D 91 (2015) 016002 [arXiv:1406.7408] [INSPIRE].

[30] J.O. Andersen, W.R. Naylor and A. Tranberg, Inverse magnetic catalysis and regularization in the quark-meson model, JHEP 02 (2015) 042 [arXiv:1410.5247] [INSPIRE].

[31] N. Mueller, J.A. Bonnet and C.S. Fischer, Dynamical quark mass generation in a strong external magnetic field, Phys. Rev. D 89 (2014) 094023 [arXiv: 1401.1647] [INSPIRE].

[32] A.N. Tawfik and N. Magdy, SU(3) Polyakov linear- $\sigma$ model in an external magnetic field, Phys. Rev. C 90 (2014) 015204 [arXiv: 1406.7488] [INSPIRE].

[33] A.N. Tawfik and N. Magdy, SU(3) Polyakov linear- $\sigma$ model in magnetic fields: Thermodynamics, higher-order moments, chiral phase structure and meson masses, Phys. Rev. C 91 (2015) 015206 [arXiv: 1501.01124] [InSPIRE].

[34] E.S. Fraga, B.W. Mintz and J. Schaffner-Bielich, A search for inverse magnetic catalysis in thermal quark-meson models, Phys. Lett. B 731 (2014) 154 [arXiv:1311.3964] [INSPIRE].

[35] T.D. Cohen and N. Yamamoto, New critical point for QCD in a magnetic field, Phys. Rev. D 89 (2014) 054029 [arXiv:1310.2234] [INSPIRE].

[36] E.M. Ilgenfritz, M. Muller-Preussker, B. Petersson and A. Schreiber, Magnetic catalysis (and inverse catalysis) at finite temperature in two-color lattice QCD, Phys. Rev. D 89 (2014) 054512 [arXiv: 1310.7876 ] [INSPIRE].

[37] J. Braun, W.A. Mian and S. Rechenberger, Delayed Magnetic Catalysis, arXiv:1412.6025 [INSPIRE].

[38] K.A. Mamo, Inverse magnetic catalysis in holographic models of QCD, JHEP 05 (2015) 121 [arXiv: 1501.03262] [INSPIRE].

[39] N. Mueller and J.M. Pawlowski, Magnetic catalysis and inverse magnetic catalysis in QCD, Phys. Rev. D 91 (2015) 116010 [arXiv: 1502.08011] [INSPIRE].

[40] T. Kojo and N. Su, The quark mass gap in a magnetic field, Phys. Lett. B 720 (2013) 192 [arXiv: 1211.7318] [INSPIRE].

[41] E.S. Fraga and A.J. Mizher, Chiral transition in a strong magnetic background, Phys. Rev. D 78 (2008) 025016 [arXiv:0804.1452] [INSPIRE]. 
[42] N. Callebaut and D. Dudal, Transition temperature(s) of magnetized two-flavor holographic QCD, Phys. Rev. D 87 (2013) 106002 [arXiv:1303.5674] [InSPIRE].

[43] V.A. Miransky and I.A. Shovkovy, Magnetic catalysis and anisotropic confinement in QCD, Phys. Rev. D 66 (2002) 045006 [hep-ph/0205348] [INSPIRE].

[44] V.A. Miransky and I.A. Shovkovy, Quantum field theory in a magnetic field: From quantum chromodynamics to graphene and Dirac semimetals, Phys. Rept. 576 (2015) 1 [arXiv: 1503.00732] [INSPIRE].

[45] Y. Aoki, Z. Fodor, S.D. Katz and K.K. Szabo, The Equation of state in lattice QCD: With physical quark masses towards the continuum limit, JHEP 01 (2006) 089 [hep-lat/0510084] [INSPIRE].

[46] S. Borsányi et al., The QCD equation of state with dynamical quarks, JHEP 11 (2010) 077 [arXiv: 1007.2580] [INSPIRE].

[47] M. Fukugita, M. Okawa and A. Ukawa, Finite Size Scaling Study of the Deconfining Phase Transition in Pure SU(3) Lattice Gauge Theory, Nucl. Phys. B 337 (1990) 181 [InSPIRE].

[48] G.S. Bali, F. Bruckmann, G. Endrödi, F. Gruber and A. Schäfer, Magnetic field-induced gluonic (inverse) catalysis and pressure (an)isotropy in QCD, JHEP 04 (2013) 130 [arXiv: 1303.1328] [INSPIRE].

[49] G. Cella, G. Curci, A. Vicere and B. Vigna, The SU(3) deconfining phase transition with Symanzik action, Phys. Lett. B 333 (1994) 457 [hep-lat/9405018] [INSPIRE].

[50] S. Borsányi, G. Endrodi, Z. Fodor, S.D. Katz and K.K. Szabo, Precision SU(3) lattice thermodynamics for a large temperature range, JHEP 07 (2012) 056 [arXiv:1204.6184] [INSPIRE].

[51] M. Lüscher, Properties and uses of the Wilson flow in lattice QCD, JHEP 08 (2010) 071 [Erratum ibid. 1403 (2014) 092] [arXiv: 1006.4518] [INSPIRE].

[52] S. Borsányi et al., High-precision scale setting in lattice QCD, JHEP 09 (2012) 010 [arXiv: 1203.4469] [INSPIRE].

[53] C. Bonati, M. D'Elia, M. Mariti, M. Mesiti, F. Negro and F. Sanfilippo, Anisotropy of the quark-antiquark potential in a magnetic field, Phys. Rev. D 89 (2014) 114502 [arXiv: 1403.6094] [INSPIRE].

[54] M. D'Elia, S. Mukherjee and F. Sanfilippo, QCD Phase Transition in a Strong Magnetic Background, Phys. Rev. D 82 (2010) 051501 [arXiv: 1005.5365] [INSPIRE].

[55] T. Vachaspati, Magnetic fields from cosmological phase transitions, Phys. Lett. B 265 (1991) 258 [INSPIRE].

[56] J.H. Applegate and C.J. Hogan, Relics of Cosmic Quark Condensation, Phys. Rev. D 31 (1985) 3037 [INSPIRE].

[57] E. Witten, Cosmic Separation of Phases, Phys. Rev. D 30 (1984) 272 [InSPIRE].

[58] J.S. Schwinger, On gauge invariance and vacuum polarization, Phys. Rev. 82 (1951) 664 [INSPIRE].

[59] G.V. Dunne, Heisenberg-Euler effective Lagrangians: Basics and extensions, hep-th/0406216 [INSPIRE].

[60] S. Ozaki, QCD effective potential with strong U(1)_em magnetic fields, Phys. Rev. D 89 (2014) 054022 [arXiv:1311.3137] [INSPIRE]. 
[61] S. Ozaki, T. Arai, K. Hattori and K. Itakura, Euler-Heisenberg-Weiss action for QCD $+Q E D$, Phys. Rev. D 92 (2015) 016002 [arXiv:1504.07532] [InSPIRE].

[62] P. Weisz, Continuum Limit Improved Lattice Action for Pure Yang-Mills Theory. 1., Nucl. Phys. B 212 (1983) 1 [inSPIRE].

[63] http://physics.utah.edu/ detar/milc.html.

[64] I. Montvay and G. Münster, Quantum fields on a lattice, Cambridge Monographs on Mathematical Physics, Cambridge University Press, Cambridge U.K. (1997). 